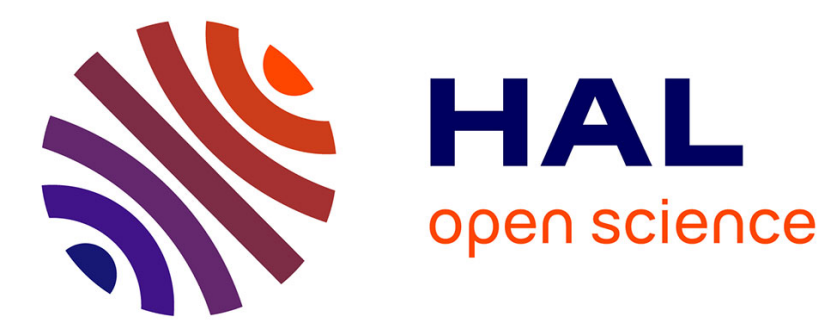

\title{
Le dynamisme interne des corpuscules et l'origine de la gravitation
}

\author{
A. Kastler
}

\section{To cite this version:}

A. Kastler. Le dynamisme interne des corpuscules et l'origine de la gravitation. J. Phys. Radium, 1931, 2 (2), pp.61-64. 10.1051/jphysrad:019310020206100 . jpa-00233051

\section{HAL Id: jpa-00233051 https://hal.science/jpa-00233051}

Submitted on 1 Jan 1931

HAL is a multi-disciplinary open access archive for the deposit and dissemination of scientific research documents, whether they are published or not. The documents may come from teaching and research institutions in France or abroad, or from public or private research centers.
L'archive ouverte pluridisciplinaire HAL, est destinée au dépôt et à la diffusion de documents scientifiques de niveau recherche, publiés ou non, émanant des établissements d'enseignement et de recherche français ou étrangers, des laboratoires publics ou privés. 


\title{
LE DYNAMISME INTERNE DES GORPUSGULES ET L'ORIGINE DE LA GRAVITATION
}

\author{
par A. KASTLER.
}

\begin{abstract}
Sommaire. - Le principe d'équivalence entre champs de gravitation et champs d'inertie suggère l'idée que ces deux sortes de champs doivent leur existence au mème mécanisme physique élémentaire.

Or, les forces d'inertie sont des forces d'origine dynamique, produites par l'accélération d'un corps par rapport à la masse d'univers. Elles ne constituent qu'un cas particulier d'un phénomène plus général : L'accélération mutuelle de deux corps crée en ceux-ci des a forces d'accélération " qui s'y opposent. Ces forces sont trop faibles pour pouvoir être décelées avec deux corps de masse usuelle; elles nous apparaissent comme « forces d'Inertie " Iorsqu'on accélère un corps par rapport à la masse considérable de tout l'univers.

Conformément au principe d'équivalence, il convient donc d'interpréter les forces de gravitation comme un autre cas particulier de forces d'accélération. L'ancienne conception statique de la gravitation doit donc faire place à une conception dynamique qui devra rechercher le dynamisme caché qui engendre l'effet de gravitation. Cet effet émane des corpuscules matériels ultimes (proton et électron) et doit donc être attribué à un dynamisme interne des corpuscules. Ce caractère dynamique des corpuscules s'est affirmé de plus en plus et de divers côtés pendant les dernières années (spin de l'électron et du proton, vibration interne de de Broglie). C'est en lui, et par les accélérations internes qu'il conditionne, qu'il convient de rechercher l'origine de la gravitation.
\end{abstract}

“ Il importe de noter que la déformation de l'Espace-Temps ne doit pas être considérée comme la cause de lá gravitation. Entre la structure de l'univers et la gravitation il n'y a pas de lien de causalité, car c'est une seule et même chose. Les phénomènes de gravitation sont simplement des manifestations de la déformation qui existe en présence ou au voisinage de la matière, qui est soumise à une loi découverte par Einstein, mais dont la cause première resle un profond myslère. ”

Jean Becquerel.

(Le Principe de Relalivité et la Théorie de la Gravitation, p. 142).

Einstein a fait faire un grand progrès au problème de la gravitation en énonçantle principe d'équivalence entre les champs de gravitation et les champs d'inertie. C'est sur ce principe qu'il a fondé sa théorie de la gravitation, théorie géométrique et formelle qui Famène l'étude de la gravitation à l'étude des propriétés d'un univers non euclidien (ce qui revient au point de vue physique à substituer dans tout domaine infinitésimal au champ de 
gravitation un champ (l'inertie équivalent). Il est évident que cette théorie, comme le fait remarquer Becquerel, ne résout pas le problème de l'origine de la gravitation, car elle ne fait qu'expliciter analytiquement le contenu physique du principe d'équivalence.

En partant de ce principe, on peut cependant essayer d'aboutir à un point de vue nouveau : il exprime en effet l'impossibilité de distinguer un champ de gravitation l'un champ d'inertie. Les deux champs ne constituent qu’une seule et mème réalité physique.

Or de l'identité de leurs propriétés il est logique de conclure à l'identité de leurs causes.

La gravitation et linertie de la matière doivent être attribuées à une méme cause physique.

Pénétrer la cause de l'une servira donc à élucider la cause de l’autre.

Que savons-nous sur l'origine des forces d'inertie? Ces forces ont une origine essentiellement dynamique : elles apparaissent avec le mourement, avec le mouvement accéléré. Toute accélération d'un corps par rapport à un système galiléen fait naître des forces d'inertie. Pourquoi les sy̌stèmes galiléens sont-ils favorisés parmi les autres, à quelle cause physique tangible peut-on attribuer leur rôle privilégié ? C'est pour répondre à cette question et pour éliminer la notion métaphysique d'espace absolu que Mach et après lui Einstein ont fait intervenir comme cause objective la masse d'univers $\left(^{1}\right)$.

C'est la masse totale de l'univers et sa répartition dans l'espace qui détermine la difiérentiation entre systèmes galiléens et systèmes non galiléens, les premiers étant ceux par rapport auxquels le « centre de gravité » de l'univers n’est pas accéléré. Il convient donc de modifier l'énoncé du principe de l'inertie, pour lui donner un sens physique et de dire:

Toute accélération relative d'un corps par rapport à la masse d'univers détermine l'apparibion de forces d'inertie qui s'opposent à cette accélération.

Cet énoncé a l'avantage de faire envisager les forces d'inertie comme un cas parliculier d'un phénomème beaucoup plus général :

L'accélération relative de deux masses détermine des “forces qui s'y opposent».

Nous voulons appeler ces forces, pour abréger, “forces d'accélération ». Ces forces sont si faibles qu'il faut renoncer à les déceler par des expériences de laboratoire (échec de l'expérience des frères Friedländer), mais lorsque l'une de ces masses est la masse tout entière de l'univers, on comprend que l'effet soit sensible, et il nous apparaît alors dans les forces que nous appelons « forces d'inertie » $\left({ }^{2}\right)$.

Ceci étant admis sur l'origine des forces d'inertie, nous allons nous en servir pour élucider le problème de l'origine de la gravitation.

Conformément à notre postulat, il nous faut rechercher l'origine de ces forces également dans l'existence d'accélérations relatives. Nous savons que Ies champs de gravitation émanent des éléments ultimes de la matière, de l'electron et surtout du proton. Du point de vue des anciennes conceptions statiques de la matière notre recherche semble vaine. L'électron et le proton y sont considérés comme des sphères isotropes, au repos, et chargées d'électricité, centres de champs électrostatiques. La matière, constituée par leur assemblage, semble engendrer autour d'elle le champ de gravitation statiquement par le fait d'ètre présente.

Cette conception statique de la gravitation, qui ne cache que notre ignorance, est incompatible avec une interprétation dynamique de la gravitation, analogue à celle des forces d'inertie.

Mais depuis quelques années l'idée d'un dynamisme interne des corpuscules s'affirme de plus en plus en Physique. Cette idée s'est imposée aux Plısiciens dans deux domaines tout à fait distincts :

(1) Ernst Mach, Die Mechanik. - Einstein, die Grindlagen der allyemeinen lielativitïtstheorie, p. 8 et 9 , - Freundlich, Grundlagen der Einsteinschen Gravitationsthéarie, p. 39 et 40.

( $\left.{ }^{2}\right)$ Einstein, Vier Vorlesungen uber Relativititstheorie, p. 64 . 
$1^{\circ}$ Pour expliquer les multiplicités spectrales, les spectroscopistes se sont vu's obliqés d'admettre l'existence d'un mouvement de rotation propre de l'électron, le spin d'Uhlenbeck et Goudsmit. L'existence de deux molécules d'hydrogène (ortho et para) semble indiquer que ce spin appartient également au proton, les deux variétés d'hydrogène se distinguant par la résultante des spins nucléaires de la molécule $\mathbf{H}^{2}$.

2. Dans un autre ordre l'idées Louis de Broglie a affirmé le dynamisme interne des corpuscules matériels par son postulat fondamental :

$$
E=m c^{2}=h \nu
$$

qui attribue à tout quantum d énergie $E$ (proton, électron ou photon) une vibration interne de fréquence.

Ce postulat qui affirme l'universalité du dynamisme interne dela matière, a été confirmé expérimentalement par la diffraction des électrons.

Dirac a montré que ces deux nouvelles propriétés des corpuscules (vibration interne et spiin) ne sont que deux aspects différents d'un mème dynamisme originel. Partant de ceci, essayons de nous faire une image concrètre de ce dynamisme si toutefois cela est possible. De Broglie ne précise en rien la nature et la forme de la vibration interne dont il postule l'existence, et son postulat ne nous permet donc pas de nous en faire un modèle mécanique. La notion de spin est mieux accessible à notre imagination, elle nous suggère de comparer les corpuscules à des sphères tournantes, véritables petits volants animés d'un mouvement de rotation extraordinairement rapide, et étant par suite le siège d'accélérations centripètes considérables. Ce dynamisme est inséparable de l'idée même decorpuscule, il en constitue une qualité première comme sa charge électrique.

Ces accélérations internes des corpuscules doivent se manifester au dehors par des forces d'accélération s'exerçant sur des corpuscules voisins et qui nous apparaissent dans les forces de gravitation.

Nous sommes donc ramenés ainsi au problème suivant : Rechercher le « champ d'accélération » produit par la rotation d'un volant, problème qui préoccupait les frères Friedländer.

Einstein, Vier Verlesüngen über Relativitätstheorie, p. 64 a traité un problème tout à fait analogue, celui du corps creux tournant. Il prévoit l'existence dans la cavité d'un. champ de Coriolis qui entraîne les corps dansle sens de la rotation, ainsi que d'un champ de forces radiales centrifuges.

Nous cherchons au contraire le champ de force produit par un corps tournant sur les corps placés à son extérieur.

Par analogie nous devons préroir autour de lui l'existence :

a) D'un champ de Coriolis cherchant à entraîner les corps dans la rotation et cherchant à supprimer ainsi l'accélération mutuelle.

b) D'un champ de force radial et centripète, le rapprochement atténuant encore l'accélération mutuelle (car dans tous les cas les forces d'accélérations cherchent à atténuer les accélérations mutuelles de matière). Considérons maintenant un morceau de matièr'te formé d'un grand nombre de corpuscules, dont les axes de rotation sont distribués au hasard.

Les forces de Coriolis auront une résultante nulle par compensation statistique, mais les forces centripètes se renforceront et leur résultante constitue à notre échelle macroscopique le champ de gravitation.

Ce champ apparait ainsi comme une résultante statistique des champs d'accélérations des corpuscules tournants.

Cette nouvelle conception de la gravitation demande évidemment à c̀tre précisée par l'analyse mathématique. Elle doit conduire, si elle est juste, à une relation numérique fai- 
sant dériver la constante de gravitation de Cavendish du quantum d'action $h$ qui régit le dynamisme interne de la matière

$$
\left(\nu=\frac{E}{h}, \quad \text { Moment cinétique de rotation }=\frac{1}{2} \frac{h}{2 \pi}\right) .
$$

Les champs de gravitation apparaissent donc comme une manifestation au dehors des accélérations internes des corpuscules; leur origine dynamique révèle ainsi leur idendité avec les forces d'inertie et fait comprendre la cause profonde du principe d'équivalence d'Einstein. 